\title{
The Impact of COVID-19 on HIV Self-Management, Affective Symptoms, and Stress in People Living with HIV in the United States
}

\author{
Rachel K. Wion ${ }^{1}\left[\right.$ ] Wendy R. Miller ${ }^{1}$ \\ Accepted: 4 June 2021 / Published online: 15 June 2021 \\ (c) The Author(s), under exclusive licence to Springer Science+Business Media, LLC, part of Springer Nature 2021
}

\begin{abstract}
COVID-19 has the potential to detrimentally impact HIV self-management in people living with HIV (PLHIV). Effective HIV-self management is critically important in managing symptoms as well as viral suppression. We examined the impact of the COVID-19 pandemic on HIV self-management, social support, social isolation, depressive symptoms, anxiety, and stress in PLHIV. 85 PLHIV were recruited from social media sites and completed an online survey. Data were collected between April 23 and 30, 2020. Participants reported increases in social isolation, depressive symptoms, anxiety, and stress and decreases in social support and overall HIV self-management from pre- to during the pandemic. Additionally, the Social Support domain and Chronic Nature of HIV domain of the HIV Self-Management Scale were also decreased from pre- to during the pandemic. The ability for PLHIV to maintain HIV self-management during this time is essential and HIV care providers should have plans in place to provide support.
\end{abstract}

Keywords HIV/AIDS $\cdot$ COVID-19 $\cdot$ Self-management $\cdot$ Affective symptoms $\cdot$ Stress

\section{Introduction}

Severe acute respiratory syndrome coronavirus 2 (SARSCoV-2), the virus that causes coronavirus disease 2019 (COVID-19), was declared a global pandemic on March 11, 2020 by the World Health Organization [1]. In the United States, by March 2020 President Trump and all 50 states had declared a state of emergency related to COVID-19 and most states had implemented some variant of stay-at-home orders in the hopes of assuaging spread of the virus [2] and preventing a surge of COVID-19 patients from overwhelming hospital systems. Social distancing and COVID-19 mitigation measures were also implemented (e.g., avoid crowds, remain at least six feet apart, work from home, and wear masks). Furthermore, during this time many healthcare clinics shut down completely or started virtual office visits to help alleviate the strain on hospital systems as well as to decrease transmission of the virus. Taken together, these measures could lead to difficulties in disease self-management for people living with chronic conditions [3-5], potentially making

Rachel K. Wion

rkwion@iu.edu

1 Indiana University School of Nursing, 600 Barnhill Dr., Indianapolis, IN 46202, USA them vulnerable to complications. Many people living with chronic conditions had their nonurgent healthcare (both physical and mental) visits canceled, had difficulty contacting their healthcare providers, and some were not able to obtain their medications due to supply issues. Others were afraid of contracting COVID-19 and chose to not engage in healthcare [6]. There is a need to determine which aspects of self-management of chronic conditions are most affected by the COVID-19 pandemic so that plans can be put into place to support people living with chronic conditions during this time.

Most people infected with COVID-19 recover and do not need to be hospitalized. Those who are most at risk for severe complications related to COVID-19 include older age and people with conditions such as serious heart conditions, chronic obstructive pulmonary disease, cancer, chronic kidney disease, Type 2 diabetes, being immunocompromised due to solid organ transplant, sickle cell disease, and having a body mass index of 30 or higher [7]. One population for whom there is concern about severe complications related to COVID-19 is people living with HIV (PLHIV) due to the possibility of them being immunocompromised if not virally suppressed. While the COVID-19 pandemic is still evolving, according to the Centers for Disease Control and Prevention (CDC), the 
risk for severe illness related to COVID-19 in PLHIV compared to the general population is unknown [7]. However, PLHIV with a low CD4 cell count, not on antiretroviral treatment (ART), or any of the previously noted chronic conditions are at higher risk for COVID-19 complications [7]. Given the novel status of the SARS-CoV-2 virus, there is a paucity of published studies on COVID-19 risk in PLHIV. In an observational prospective study conducted in Spain, $1.8 \%(N=51)$ of all PLHIV followed by an HIV clinic in a large university hospital were either infected or suspected to be infected with COVID-19 [8]. Of those, $75 \%$ had a case that was considered mild to moderate, while $25 \%$ had a severe case. Over half (55\%) were hospitalized, $12 \%$ were admitted to intensive care, and $4 \%$ died. Most of the PLHIV had at least one comorbid condition [8]. These results are consistent with an observational retrospective study conducted in an Italian hospital [9]. 47 PLHIV had confirmed or probable COVID-19. Of those, $28 \%$ were hospitalized, $4 \%$ died, and $64 \%$ had a least one comorbid condition. The recovery time was 14 days $(S D=8)$ after onset of symptoms [9]. Conclusions about the severity of COVID-19 illness in PLHIV drawn from these studies are limited due to small sample sizes and single-center study sites. A multi-site study examined all PLHIV receiving ART in HIV clinics associated with 60 hospitals in Spain over a 1.5-month period [10]. Out of 77,590 PLHIV, 236 were diagnosed with COVID-19. For the PLHIV diagnosed with COVID-19, 64\% needed to be hospitalized, $6 \%$ were admitted to intensive care, and $8 \%$ died. However, compared to the general Spanish population with the same age range, the risks for COVID-19 and death from COVID-19 were similar. In PLHIV the risk for COVID-19 was 30.0 per 10,000 people and the risk for death was 3.7 per 10,000 . In the general population, the risk for COVID-19 was 33 per 10,000 people (after excluding health care workers) and risk for death was 2.1 per 10,000 [10]. A limitation of this study was not controlling or accounting for comorbid conditions.

For successful care outcomes related to HIV, it is important that PLHIV remain both connected and engaged in the HIV care continuum which, after diagnosis, linkage to, and receipt of medical care, includes remaining in medical care and achieving and maintaining viral suppression [11]. HIV self-management includes practices that promote the successful outcomes outlined by the HIV care continuum such as communicating with healthcare providers as needed, keeping clinic appointments, and taking ART as prescribed. Also important to HIV self-management are other aspects of health such as HIV symptom management (e.g., fatigue, depressive symptoms, anxiety, and problems with sleep $[12,13])$, physical activity, eating a healthy diet, working to reach health goals, taking personal time, reducing stress, and seeking social support
[14]. The inability to effectively self-manage HIV can lead to disruptions in healthcare engagement and medication adherence which can affect viral suppression of HIV [11].

A few studies have examined how certain aspects HIV self-management have been impacted by COVID-19 in PLHIV. Kalichman et al. [6] investigated protective behaviors related to COVID-19 in 162 PLHIV who were currently participating in a longitudinal study in Atlanta, Georgia and found that $19 \%$ of the sample had missed an HIV health care appointment in the past 30 days and nearly half of those (45\%) attributed their missing appointment to the pandemic. However, ART adherence had improved over a 1-month period [6]. Similarly, Algarin et al. [15] examined factors related to COVID-19 in 16 older PLHIV who were enrolled in a mind-body intervention feasibility study. They found all participants were able to maintain ART use and most (83.3\%) kept their HIV health care appointments and maintained contact with their HIV case manager. Participants' mean stress rating related to social distancing was $4.4(S D=3.3)$ on a $1-10$ scale with higher numbers indicating greater stress [15]. Finally, Santos et al. [16] examined factors related to COVID-19 in a large international sample $(N=3732)$ of gay men and other men who have sex with men who use the Hornet social media app. They included a subset of participants who were living with HIV $(n=473)$. Most were able to obtain their ART medications; however, $18 \%$ were unable or had difficulty accessing these medications. Almost a quarter (23\%) lost access to their health care providers. Participants who lost their health insurance were more likely to report a loss of health care provider access when compared with those who maintained health insurance [16].

PLHIV have been identified as being likely to experience treatment disruptions during the COVID-19 pandemic [17]. Given the importance of HIV self-management to positive care outcomes and quality of life, it is critical to determine how PLHIV handle their self-management during the COVID-19 pandemic and what support they may need. Furthermore, social isolation related to social distancing recommendations may be harmful to mental health [17] so this should be examined as well. Much is still unknown about how COVID-19 has influenced all areas of HIV self-management in PLHIV; therefore, the purpose of this study was to examine how the COVID-19 pandemic has impacted HIV self-management, social support, social isolation, depressive symptoms, anxiety, and stress. The CDC has indicated that SARS-CoV-2 may present in waves so findings from this study will be important in determining which aspects of HIV self-management to focus on when supporting PLHIV during this time and potential future waves. 


\section{Methods}

\section{Study Design and Sample}

This study was cross-sectional and used an online survey approach. The study was deemed exempt by the Indiana University Institutional Review Board. Participants were recruited from Facebook, Instagram, and Twitter. A paid advertisement was used for Facebook using the "boost post" function. The advertisement was targeted to people who were age 18 and older, lived in the United States, and had an interest in epidemiology of HIV/AIDS. A paid advertisement was used as well for Instagram and was targeted to men and women age 18 and older, living in the United States, with an interest in the epidemiology of HIV/AIDS or HIV/AIDS research. The study was advertised on Twitter using hashtags, \#HIV and \#COVID19. These hashtags were used on Instagram as well. The study advertisement is displayed in Supplementary Material Appendix A. Inclusion criteria for the study was age 18 and older and having an HIV diagnosis. Potential participants answered two screening questions which included eliminating participants who were HIV-negative. The screening questions asked if the potential participants were age 18 or older and whether they had been diagnosed with HIV.

\section{Measures}

The survey was comprised of 64 items. Demographic items measured age, race, demographic region, education level, income, employment status (both pre-pandemic and selfreported change in employment status due to the pandemic), gender, sexual orientation, and relationship status. The following health characteristics were collected: chronic conditions, smoking status, CD4 cell count, HIV viral load, and ART medication use. The health characteristics were based on participants' status at the time of data collection.

\section{Factors Related to the COVID-19 Pandemic}

Several items measured factors related to the COVID-19 pandemic. Some of the items assessed certain factors without asking for pre-pandemic comparisons (e.g., social distancing, financial worries). Other items measured factors based on how the participants perceived them pre-pandemic and during the pandemic (e.g., overall HIV self-management, social support).

\section{Ratings of COVID-19 Pandemic Factors without Pre-Pandemic Comparisons}

Participants rated worries related to the impact of COVID19 on their financial health and their physical health on a scale from 0 to 10 with $0=$ not at all and $10=$ very much.
Participants also rated their degree of worry for the health and safety of their own self, close family, friends, local community, and society at large during the pandemic on a ten-point Likert scale from 0 to 10 with $0=$ low and $10=$ high. Additionally, participants rated the degree to which they were following social distancing guidelines and the degree to which their HIV status influenced their engagement in social distancing on a scale of $0-10$ with $0=$ not at all and $10=$ completely. Participants also rated the degree to which they thought their HIV status put them at a higher risk for experiencing a more severe COVID-19 infection if they contracted COVID-19 on a 0-10 scale with $0=$ not at all and $10=$ very much.

Participants rated whether the COVID-19 pandemic changed their ability to communicate with their healthcare providers about HIV-related concerns and their ability to resolve HIV-related problems. These were scored on a $0-10$ Likert scale with $0=$ no change and $10=$ much more difficult. Finally, participants rated their confidence in their ability to get timely help for an HIV-related problem from their HIV care providers, their local hospital, and their local pharmacy, if needed. These were scored on a $0-10$ Likert scale with $0=$ not at all confident and $10=$ extremely confident.

Finally, participants were asked whether aspects of their HIV self-management were made more difficult due to the COVID-19 pandemic. These 12 domains included the ability to: exercise, manage affective symptoms, maintain a social support network, manage stress, eat a healthy diet, reach healthcare providers, get an adequate amount of sleep, get medications from a pharmacy when needed, manage HIV medication side effects, take HIV medications as prescribed, and manage HIV symptoms. Participants were to rate each of the 12 aspects as either much more difficult, somewhat more difficult, a little bit more difficult, or not at all more difficult due to the COVID-19 pandemic. They were not asked to report on how they self-managed these domains prior to the pandemic.

\section{Ratings of COVID-19 Pandemic Factors with Pre-Pandemic Comparisons}

Given the widespread social distancing orders and recommendations and how these may affect mental health, participants were asked questions related to social support and social isolation. Participants were asked to rate their social support network prior to the COVID-19 pandemic and during the pandemic on a Likert scale from 0 to 10 with $0=$ very limited and $10=$ very strong. Similarly, participants were queried regarding how often they felt socially isolated preand during the pandemic on a Likert scale from 0 to 10 with $0=$ never and $10=$ very often. 
HIV Self-Management HIV self-management was measured using the HIV Self-Management Scale [14]. The HIV Self-Management Scale is a 20-item measure that is comprised of three domains-Daily Self-Management Health Practices (12 items), Social Support and HIV SelfManagement (3 items), and Chronic Nature of HIV SelfManagement (5 items). The HIV Self-Management Scale was administered twice. First, participants were asked how often the items applied to them prior to the COVID-19 pandemic. Then, participants were queried about how often the items applied to them during the pandemic. Participants were asked to respond to the items on a Likert scale from 0 to 3 with $0=$ not applicable, $1=$ none of the time, $2=$ some of the time, and 3=all of the time. The HIV Self-Management Scale is not reported using a composite score for the entire scale, rather, a composite score for each of the three domains is reported separately with higher scores indicating greater HIV self-management. Scores range from 0-36 for the Daily Self-Management Health Practices domain, 0-9 for the Social Support and HIV Self-Management domain, and 0-15 for the Chronic Nature of HIV Self-Management domain. Originally conceptualized as a measure of HIV self-management in women [14], the HIV Self-Management Scale has also been used in a mixed gender study with acceptable reliability [18].

Participants were also asked to rate their ability to handle their overall HIV self-management (e.g., medications, health provider visits, mental health, healthy diet, exercise) pre- and during the pandemic on a 0-10 Likert scale with $0=$ unable to do it and $10=$ very well.

Depressive Symptoms Depressive symptoms were measured using the Patient Reported Outcomes Measurement Information System (PROMIS) Item Bank v1.0-Emotional Distress-Depression-Short Form 8a instrument [19]. This instrument was administered twice. First, the participants were asked to respond based on their symptoms pre-pandemic and then they were to answer based on their symptoms during the COVID-19 pandemic. Participants were asked to respond to eight statements on a Likert scale from 1 to 5 with $1=$ never and $5=$ always. Scores range from 8 to 40 and higher scores are indicative of greater depressive symptoms.

Anxiety Anxiety was measured using the PROMIS Item Bank v1.0_Emotional Distress-Anxiety-Short Form 8a instrument [19]. As with depressive symptoms, this instrument was administered twice. First, the participants were asked to answer based on their symptoms pre-pandemic and then they were to respond based on their symptoms during the pandemic. Participants were asked to respond to eight statements on a Likert scale from 1 to 5 with $1=$ never and
$5=$ always. The scores range from 8 to 40 with higher scores indicative of greater anxiety.

Stress Perceived stress was measured using the NIH Toolbox ${ }^{\circledR}$ Item Bank/Fixed Form v2.0_-Perceived Stress (Ages $18+$ ) [20]. This instrument was administered twice. Participants were first asked to respond to the items based on their perceived stress pre-pandemic and then during the pandemic. There were ten items that were scored on a Likert scale from 1 to 5 with $1=$ never and $5=$ very often. Four of the items were reversed scored. Scores range from 10 to 50 with higher scores indicating greater stress.

\section{Data Collection}

The survey was administered online via the Qualtrics platform from April 23 to April 30, 2020. After viewing the study advertisement, potential participants could determine if they were eligible for the study by clicking on a link to the survey. They first answered the screening questions and if they met the criteria, the participants were then directed to the survey items. The survey took approximately 20-30 min to complete. All participants were given the option to be placed in a drawing for one of two $\$ 50$ gift cards.

\section{Analysis}

Descriptive statistics were conducted. Descriptive analyses were also performed to determine any mean differences in HIV self-management, social support, social isolation, depressive symptoms, anxiety, and stress during COVID19 based on participants' age, race, and years living with HIV. Paired t-tests were performed to determine whether there were any significant mean differences in HIV self-management, social support, social isolation, depressive symptoms, anxiety, and stress, pre- and during the pandemic. All paired t-test assumptions were tested and extreme outliers (i.e., more than three box-lengths away from the edge of their box in the boxplot) were removed prior to analysis. The data were analyzed using SPSS version 26 and version 27 software.

\section{Results}

A total of 154 participants accessed the study, met the screening criteria to participate in, and started the study. 85 participants remained for analysis in the final sample. Participants were eliminated due to incomplete submissions (i.e., those who completed less than $90 \%$ of the items; $n=67$ ) and full submissions that were completed too quickly (i.e., less than $5 \min , n=2$ ). 


\section{Demographic Characteristics}

Sample characteristics are displayed in Table 1. The mean age of the participants was 49.9 years $(S D=12.5$; range $26-76$ years). The participants were largely men $(95.3 \%$, $n=81)$, white $(64.7 \%, n=55)$, gay or lesbian $(84.7 \%$, $n=72$ ), and married or in a long-term relationship $(48.2 \%$, $n=41)$. Most of the sample had achieved a bachelor's degree or higher $(49.4 \%, n=42)$, were employed full-time pre-pandemic $(56.5 \%, n=48)$, and had an annual household income of less than $\$ 60,000(64.8 \%, n=55)$. All nine regions of the United States [21] were represented with the majority $(57.7 \%, n=49)$ living in the Pacific (e.g., Alaska, California, Hawaii, Oregon, and Washington; 22.4\%, $n=19$ ), East North Central (e.g., Illinois, Indiana, Michigan, Ohio, and Wisconsin; 18.8\%, $n=16$ ), and South Atlantic (e.g., Delaware, Florida, Georgia, Maryland, North Carolina, South Carolina, Virginia, District of Columbia, and West Virginia; 16.5\%, $n=14$ ) areas. Participants were also from the Mid-Atlantic (New Jersey, New York, and Pennsylvania; 11.8\%, $n=10$ ), West South Central (Arkansas, Louisiana, Oklahoma, and Texas; $10.6 \%, n=9$ ), East South Central (Alabama, Kentucky, Mississippi, and Tennessee; 7.1\%, $n=6$ ), New England (Connecticut, Maine, Massachusetts, New Hampshire, Rhode Island, and Vermont; 4.7\%, $n=4$ ), Mountain (Arizona, Colorado, Idaho, Montana, Nevada, New Mexico, Utah, and Wyoming; 4.7\%, $n=4$ ), and West North Central (Iowa, Kansas, Minnesota, Missouri, Nebraska, North Dakota, and South Dakota; $2.4 \%, n=2$ ) regions. One participant did not report their location.

\section{Health Characteristics}

Health characteristics are reported in Table 1. Most participants had been living with HIV for at least 6 years $(75.3 \%, n=64)$ with a mean of 16.5 years $(S D=11.4$; range 1-38 years). The majority had a CD4 cell count of at least 201 cells $/ \mathrm{mm} 3(89.5 \%, n=76)$ and almost all were virally suppressed $(94.1 \% ;<200$ copies $/ \mathrm{mL}, n=80)$. All but one participant reported currently taking ART medications. Several participants reported being diagnosed with a chronic health condition with depression $(36.5 \%, n=31)$, anxiety $(35.3 \%, n=30)$, and hypertension $(34.1 \%, n=29)$ being the most frequent.

\section{Factors Related to the COVID-19 Pandemic}

The COVID-19 pandemic had impacted several different aspects of the participants' lives. At the time of data collection, $88.2 \%(n=75)$ of participants reported living in an area where the distancing orders were such that businesses were mostly closed with only those deemed essential being open. Another $10.6 \%(n=9)$ reported partial closures with some non-essential
Table 1 Sample descriptions of demographic and health characteristics $(N=85)$

\begin{tabular}{|c|c|}
\hline & $n(\%) / \operatorname{Mean}(\mathrm{SD})$ \\
\hline Age & $49.9(12.5)^{\mathrm{a}}$ \\
\hline \multicolumn{2}{|l|}{ Race/Ethnicity } \\
\hline White & $55(64.7)$ \\
\hline Latinx & $13(15.3)$ \\
\hline Black & $9(10.6)$ \\
\hline Other & $12(14.1)$ \\
\hline \multicolumn{2}{|l|}{ Gender } \\
\hline Man & $81(95.3)$ \\
\hline Woman & $3(3.5)$ \\
\hline Non-binary & $1(1.2)$ \\
\hline \multicolumn{2}{|l|}{ Education } \\
\hline Less than high school & $3(3.5)$ \\
\hline High school diploma/GED & $6(7.1)$ \\
\hline Postsecondary education & $33(38.8)$ \\
\hline Bachelor's degree or higher & $42(49.4)$ \\
\hline \multicolumn{2}{|l|}{ Income } \\
\hline Less than $\$ 20,000$ & $18(21.2)$ \\
\hline$\$ 20,000-\$ 39,999$ & $23(27.1)$ \\
\hline$\$ 40,000-\$ 59,999$ & $14(16.5)$ \\
\hline$\$ 60,000$ and above & $30(35.2)$ \\
\hline \multicolumn{2}{|l|}{ Employment status (prior to COVID-19) } \\
\hline Employed full-time & $48(56.5)$ \\
\hline Employed part-time & $11(12.9)$ \\
\hline On disability & $12(14.1)$ \\
\hline Retired & $9(10.6)$ \\
\hline Unemployed, looking for work & $3(3.5)$ \\
\hline Unemployed, not looking for work & $1(1.2)$ \\
\hline Student & $1(1.2)$ \\
\hline \multicolumn{2}{|l|}{ Sexual orientation } \\
\hline Gay or lesbian & $72(84.7)$ \\
\hline Bisexual & $7(8.2)$ \\
\hline Other & $5(5.9)$ \\
\hline \multicolumn{2}{|l|}{ Relationship status } \\
\hline Single, never married & $35(41.2)$ \\
\hline Partnered, long-term & $21(24.7)$ \\
\hline Married & $20(23.5)$ \\
\hline Widowed & $7(8.2)$ \\
\hline Divorced & $2(2.4)$ \\
\hline Years living with HIV & $16.54(11.4)^{\mathrm{b}}$ \\
\hline Currently taking ART & $84(98.8)$ \\
\hline HIV viral load $<200$ copies/mL & $80(94.1)$ \\
\hline \multicolumn{2}{|l|}{ CD4 T-cell count, cells $/ \mathrm{mm}^{3}$} \\
\hline$\leq 200$ & $2(2.4)$ \\
\hline $201-499$ & $19(22.4)$ \\
\hline$\geq 500$ & $57(67.1)$ \\
\hline Current smoker/vape user & $19(22.4)$ \\
\hline \multicolumn{2}{|l|}{ Diagnosed comorbidities } \\
\hline Depression & $31(36.5)$ \\
\hline Anxiety & $30(35.3)$ \\
\hline
\end{tabular}


Table 1 (continued)

\begin{tabular}{lc}
\hline & $n(\%) /$ Mean $(\mathrm{SD})$ \\
\hline Hypertension & $29(34.1)$ \\
Diabetes & $10(11.8)$ \\
Asthma & $9(10.6)$ \\
Heart disease & $8(9.4)$ \\
COPD & $5(5.8)$ \\
\hline
\end{tabular}

Not all variables sum to total $N$ due to missing data or more than one response option (e.g., race/ethnicity and diagnosed comorbidities)

$A R T$ antiretroviral therapy, GED General Education Development

${ }^{\text {a }}$ Observed range 26-76

${ }^{\mathrm{b}}$ Observed range 1-38

businesses open. Participants were asked whether they had experienced a change in employment as a direct result of the pandemic and 30.6\% $(n=26)$ of the participants self-reported a change in work status. The 26 participants' self-reported changes in employment status were: employed full-time $(7.7 \%, n=2)$, employed part-time $(7.7 \%, n=2)$, unemployed and looking for work $(15.4 \%, n=4)$, unemployed and not looking for work $(26.9 \%, n=7)$, unable to work $(15.4 \%, n=4)$, or for other reasons $(26.9 \%, n=7)$ such as not working per their physician's orders $(n=2)$, lack of freelance or contract work $(n=3)$, and being furloughed $(n=2)$. Participants' employment status prior to the pandemic is displayed in Table 1.

Most participants reported never having symptoms of COVID-19 nor being tested for the virus $(90.6 \%, n=77)$. The eight (9.4\%) participants who either had COVID-19 symptoms or tests had the following characteristics: one reported a positive test for COVID-19; three had symptoms but did not get tested; two had symptoms, were tested, and were awaiting their results; and two did not have symptoms, were tested, and were awaiting their results.

A little over a quarter of the sample $(28.2 \%, n=24)$ reported reaching out to their healthcare providers for information about how COVID-19 could affect their health. Approximately half of the participants $(52.9 \%, n=45)$ reported that their healthcare providers gave them information on how COVID-19 could affect their health.

A small percentage $(16.5 \%, n=14)$ of the sample reported attending HIV-focused support groups and half of those $(n=7)$ indicated that they were no longer able to attend the groups because of the pandemic. The other half $(n=7)$ were able to attend the support groups either virtually or via phone.

\section{COVID-19 Pandemic Factors without Pre-Pandemic Comparisons Ratings}

Participants were moderately worried about the financial impact of the pandemic $(M=6.61, S D=2.88)$. Similarly, they were moderately worried about the impact of COVID19 on their physical health $(M=6.86, S D=2.74)$. Participants had a moderately high level of worries related to the impact of the pandemic on their self $(M=7.12, S D=2.62)$, close family $(M=7.77, S D=2.34)$, friends $(M=7.06$, $S D=2.70)$, their local community $(M=7.01, S D=2.30)$, and society at large $(M=7.81, S D=1.95)$.

Participants had high adherence to following social distance guidelines $(M=8.92, S D=1.36)$ and their HIV status moderately influenced their engagement in social distancing $(M=6.42, S D=3.40)$. Participants also perceived that their HIV status put them at a moderately higher risk for experiencing a more severe COVID-19 infection $(M=7.12$, $S D=2.88$ ).

Participants reported low moderate difficulty in their ability to communicate with healthcare providers about HIV related concerns $(M=3.00, S D=3.02)$ and low difficulty in their $(M=2.07, S D=2.54)$ ability to resolve HIV-related problems with their providers.

Finally, participants had moderately high confidence that they could get timely help for a HIV-related problem from their HIV care providers $(M=7.74, S D=2.29)$ and pharmacy $(M=7.94, S D=1.99)$ if needed. They were also moderately confident in obtaining timely help from their local hospital $(M=6.08, S D=2.90)$.

All 12 aspects of HIV self-management were affected in differing degrees of difficulty during the pandemic (Fig. 1). The top five domains that were made much more difficult were ability to exercise (30.6\%), manage affective symptoms (24.7\%), maintain their social support network (24.7\%), manage stress (23.5\%), and eat a healthy diet (17.6\%). When examining the domains that were made any degree more difficult during the pandemic (much more, somewhat, a little bit) the top five were ability to exercise (78.9\%), manage stress (76.5\%), maintain a social support network (71.8\%), get an adequate amount of sleep (64.7\%), and manage affective symptoms (64.7\%). It is important to note that there were more participants (8-16\%) who did not complete the item measuring the 12 HIV self-management aspects when compared to other items in the survey. This lower completion rate may be due to it being a drag and drop item in the Qualtrics survey (i.e., participants were instructed to drag each of the twelve aspects HIV self-management to the corresponding box based on how impacted it was by the COVID-19 pandemic). A few of the participants commented about having difficulty completing this item when using a mobile device.

\section{COVID-19 Pandemic Factors with Pre-Pandemic Comparisons Ratings}

Results for this section are displayed in Table 2. Prior to the pandemic the mean social support score was moderately 
Ability to exercise

Ability to manage affective symptoms

Ability to maintain social support network

Ability to manage stress

Ability to eat a healthy diet

Ability to reach healthcare providers

Ability to get an adequate amount of sleep

Ability to get meds from pharmacy when needed

Ability to manage HIV medication side effects

Ability to take HIV medications as prescribed

Ability to manage HIV symptoms

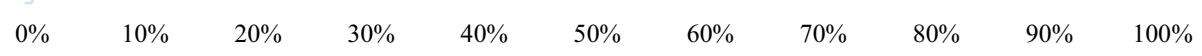

Much more difficult $\square$ Somewhat more difficult $\square$ A little bit more difficult $\quad$ Not at all more difficult $\quad$ Does not apply $\quad$ Did not answer

Fig. 1 Aspects of HIV self-management made more difficult by the COVID-19 pandemic

high $(M=7.42, S D=2.13)$ and during it was moderate $(\mathrm{M}=6.09, S D=2.93)$ a difference of $1.33(95 \% \mathrm{CI}, 0.826$, 1.832), $t(84)=5.26, p<0.001, d=0.57$. Similarly, there was an increase in social isolation from pre- $(M=3.89$, $S D=2.64)$ to during the pandemic $(M=6.14, S D=2.83)$, a difference of $-2.25(95 \% \mathrm{CI},-2.864,-1.636), t(83)=-7.29$, $p<0.001), d=0.80$.

HIV Self-Management There were no significant differences in the Daily Self-Management Health Practices domain of the HIV Self-Management Scale pre- $(M=22.73$, $S D=4.56)$ and during the pandemic $(M=22.56, S D=5.62)$, $t(83)=0.36, p=0.723$. There was a decrease in the Social Support domain from pre- $(M=3.43, S D=2.56)$ to during the pandemic $(M=2.98, S D=2.66)$, a difference of 0.45 (95\% CI, 0.245, 0.803), $t(82)=3.66, p<0.001, d=0.17$. Similarly, there was a decrease in the Chronic Nature of HIV domain from pre- $(M=13.35, S D=1.60)$ to during the pandemic $(M=13.02, S D=1.89)$, a difference of $0.33(95 \%$ CI, 0.070, 0.581), $t(82)=2.53, p<0.05, d=0.28$.

Participants reported being better at managing their overall HIV self-management prior to the pandemic $(M=8.40, S D=1.63)$ than during it $(M=7.13, S D=2.00)$,

Table 2 Descriptive statistics and t-test results for COVID-19 factors pre- and during the pandemic

\begin{tabular}{|c|c|c|c|c|c|c|c|c|c|}
\hline \multirow[b]{2}{*}{ Outcome } & \multicolumn{2}{|c|}{ Pre-pandemic } & \multicolumn{2}{|c|}{ During pandemic } & \multirow[b]{2}{*}{$\mathrm{n}$} & \multirow[b]{2}{*}{ Mean Difference $(95 \%$ CI) } & \multirow[b]{2}{*}{$\mathrm{t}$} & \multirow[b]{2}{*}{ df } & \multirow[b]{2}{*}{ d } \\
\hline & M & SD & M & SD & & & & & \\
\hline Social isolation & 3.89 & 2.64 & 6.14 & 2.83 & 84 & $-2.25(-2.864,-1.636)$ & $-7.29 * *$ & 83 & 0.80 \\
\hline Social support & 7.42 & 2.13 & 6.09 & 2.93 & 85 & $1.33(0.826,1.832)$ & $5.26 * *$ & 84 & 0.57 \\
\hline Overall HIV self-management & 8.40 & 1.63 & 7.13 & 2.00 & 85 & $1.27(0.872,1.669)$ & $6.34 * *$ & 84 & 0.69 \\
\hline $\begin{array}{l}\text { Daily Self-Management Health } \\
\text { Practices domain }^{\mathrm{a}}\end{array}$ & 22.73 & 4.56 & 22.56 & 5.62 & 84 & $0.17(-0.764,1.098)$ & 0.36 & 83 & 0.04 \\
\hline Social Support domain ${ }^{\mathrm{a}}$ & 3.43 & 2.56 & 2.98 & 2.66 & 83 & $0.45(0.245,0.803)$ & $3.66^{* *}$ & 82 & 0.17 \\
\hline Chronic Nature of HIV domain ${ }^{a}$ & 13.35 & 1.60 & 13.02 & 1.89 & 83 & $0.33(0.070,0.581)$ & $2.53 *$ & 82 & 0.28 \\
\hline Depressive symptoms & 16.17 & 6.34 & 18.17 & 8.16 & 81 & $-2.0(-3.193,-0.807)$ & $-3.34 * *$ & 80 & 0.37 \\
\hline Anxiety & 17.20 & 6.92 & 20.20 & 8.16 & 80 & $-3.0(-4.511,-1.489)$ & $-3.95 * *$ & 79 & 0.44 \\
\hline Stress & 25.60 & 6.03 & 27.52 & 6.91 & 81 & $-1.91(-2.915,-3.801)$ & $-3.80 * *$ & 80 & 0.42 \\
\hline
\end{tabular}

$* p<0.05, * * p<0.001$

${ }^{a}$ HIV Self-Management Scale 
a difference of 1.27 (95\% CI, 0.872, 1.669), $t(84)=6.34$, $p<0.001, d=0.69$.

Affective Symptoms There was an increase in depressive symptoms from pre-pandemic $(M=16.17, S D=6.34)$ to during the pandemic $(M=18.17, S D=8.16)$ a difference of -2.0 (95\% CI, $-3.193,-0.807), t(80)=-3.34, p<0.001)$, $d=0.37$ ). Similarly, there was an increase in anxiety symptoms from pre-pandemic $(M=17.20, S D=6.92)$ to during the pandemic $(M=20.20, S D=8.16)$, a difference of -3.0 $(95 \% \mathrm{CI},-4.511,-1.489), t(79)=-3.95, p<0.001$, $d=0.44$.

Stress There was an increase in stress from pre-pandemic $(M=25.60, S D=6.03)$ to during the pandemic $(M=27.52$, $S D=6.91)$, a difference of $-1.91(95 \% \mathrm{CI},-2.915$, $-3.801) t(80)=-3.80, p<0.001, d=0.42$.

\section{Discussion}

The purpose of this study was to determine how the COVID19 pandemic has influenced HIV self-management, social support, social isolation, depressive symptoms, anxiety, and stress in PLHIV. We found that the pandemic impacted all aspects of HIV self-management to varying degrees. Participants reported significantly reduced social support and social isolation from pre- to during the pandemic. Similarly, their overall HIV self-management was significantly better pre-pandemic as well as the Social Support and Chronic Nature of HIV domains of the HIV Self-Management Scale. Additionally, there was an increase in depressive symptoms, anxiety, and stress during the pandemic when compared to pre-pandemic.

The findings from this study should be considered alongside some important limitations. This study was limited by the cross-sectional design when measuring pre- and duringevent behaviors and attitudes which could cause recall bias. Future investigations should consider a longitudinal design to determine how HIV self-management needs change over time. Furthermore, current prospective cohort studies involving PLHIV should consider adding COVID-19 and HIV selfmanagement specific instruments so that changes in these can be measured temporally during the pandemic. Another limitation is that while some of the mean differences from pre- to during the pandemic may be statistically significant, they may not be clinically significant or relevant to practice. This may be because most of the variables are self-reported perceptions (e.g., social isolation, depressive symptoms) and rather than actual behaviors (e.g., some items within the HIV Self-Management Scale such as eating a healthy diet). An additional limitation is that the data were collected early in the pandemic (end of April 2020) and at this point in time, stay at home orders and social distancing recommendations varied widely across the United States in terms of when they were implemented, if at all. This may have been too early to see the full impacts of these orders on healthcare access and missed appointments for some of our participants. Future studies should examine how the COVID-19 pandemic mitigation orders impacted healthcare access at various points in time across the pandemic. Another limitation is that the online nature of recruitment could lead to an increased possibility of participants not truly meeting inclusion criteria (e.g., age less than 18 years, no HIV diagnosis). Populationtargeted social media advertisements were made to mitigate the possibility of a participant not meeting the inclusion criteria; however, there is still a chance for a participant not meeting the criteria to be included. Additionally, the sample was small $(N=85)$, largely male, and white which limits the generalizability of the findings to broader populations. Future investigations should focus on obtaining a larger and more diverse sample as certain subpopulations may be more negatively affected by the current and future pandemics.

The participants were at least moderately worried about their health in the face of the pandemic. The mean score for whether they thought they had a chance for a more severe COVID-19 infection if they contracted the virus was between moderate to high. Subsequently, the mean score for how strictly they were following social distancing guidelines indicated they were taking social distancing seriously. This diligence in social distancing is consistent with the Kalichman et al. [6] finding that almost all of their sample were following the recommended social distancing guidelines. The adherence to social distancing guidelines along with canceled healthcare appointments and inability to get medications are cause for concern that there may be some delays in HIV care. Some of the participants indicated that the COVID-19 pandemic had made their ability to contact healthcare providers and get medications from the pharmacy more difficult. These findings are consistent with the Santos et al. [16] outcomes of loss of access to HIV health care providers and increased difficulty in obtaining ART meds. This disruption to the HIV care continuum could lead to decreased HIV viral suppression [11]. However, the mean score for ability to communicate with their HIV healthcare providers indicated that the participants largely had little to no difficulty with this. Additionally, the confidence in their ability to access timely care from their HIV providers was high. Taken together these findings indicate that while some PLHIV were having treatment delays, the majority were able to engage in care if needed.

The participants' HIV self-management was adversely affected by the pandemic. Their overall HIV self-management was better managed prior to the pandemic than during it. Additionally, the Social Support and Chronic Nature of HIV (i.e., priority in managing HIV, relationship with HIV 
care provider, ability to make healthcare appointments as needed to manage various aspects of HIV) domains of the HIV Self-Management Scale were rated better prior to the pandemic. However, these results, while statistically significant are tempered by whether they are also clinically significant. Overall HIV self-management had a medium effect size but a 1.27 mean difference on a $0-10$ scale may not indicate a clinically meaningful decrease. Both the Social Support and Chronic Nature of HIV domains of the HIV Self-Management Scale had small effect sizes with very little mean differences relative to the range of their scores (0-9 for Social Support and 0-15 for Chronic Nature).

The aspects of HIV self-management that were more frequently made difficult by the pandemic included the ability to eat a healthy diet, exercise, manage stress, maintain a social support network, get an adequate amount of sleep, and manage affective symptoms. It is encouraging that HIV self-management factors like the ability to take HIV medications as prescribed and reach healthcare providers were not ranked at the top of this list, which is consistent with the findings noted earlier. However, it is concerning that certain features of HIV self-management such as exercise and sleep are ranked high on this list. Exercise has been linked to mental health indicators such as decreased depressive symptoms and anxiety [22], increased quality of life [23], and increased ART adherence [24] in PLHIV. Similarly, poor sleep has been associated with increased depressive symptoms and anxiety, and decreased ART adherence [25].

In addition to being ranked high on the list of HIV selfmanagement factors that were made more difficult by the pandemic, participants also had increased depressive symptoms, anxiety, and stress during the pandemic when compared to pre-pandemic. Again, while these were all statistically significant, it is questionable whether they are clinically significant. The effect sizes for all three variables were small. Also, the mean differences from pre- to during the pandemic were small. It is difficult to determine whether a two- or three-point increase in depressive symptoms and anxiety, respectively, is truly clinically meaningful. Greater affective symptoms and stress can lead to decreased HIV self-management and outcomes such as viral non-suppression. For instance, suboptimal ART adherence has been linked to depressive symptoms [24, 26, 27], anxiety [28], and stress [29]. Additionally, decreased retention in HIV care is associated with depressive symptoms [27].

The ability to maintain a social support network was ranked as one of the top HIV self-management factors that was negatively affected by the COVID-19 pandemic. Furthermore, participants' strength of social support networks was decreased, and feelings of social isolation were increased from pre- to during the pandemic. Similar to previous sections, this perceived decrease in social support and increase in feelings of isolation may not be clinically significant. Social support had a medium effect size along with a 1.33 mean decrease from pre- to during the pandemic. However, this did decrease them from moderately high to moderate social support on the 0-10 scale. Social support may be clinically meaningful. There was a large effect size and an over two-point increase in social isolation from preto during the pandemic. The negative impacts of social isolation and support may, in part, account for the increase in depressive symptoms and anxiety during the pandemic. Indeed, this is consistent with the literature where higher social support has been linked to a decrease in depressive symptoms [30, 31] and anxiety in PLHIV [30]. As previously noted, increases in depressive symptoms can lead to decreased HIV self-management. This is further confirmed as lower social support has been associated with decreased ART adherence [32, 33].

COVID-19 has had a negative impact on PLHIV. While certain aspects of HIV self-management such as ability to take ART medications were less affected, others, such as ability to maintain social support, were more affected. Additionally, there were increases in affective symptoms and stress in this population. While it is important that PLHIV remain engaged in healthcare and are adherent to ART, it is also critical that their emotional and mental health needs are met. HIV care providers should have a plan in place to offer support for all aspects of HIV self-management during a pandemic. The execution of this plan could be virtual or by phone and facilitated by HIV care coordinators. Further, the participants reported moderately high worries related to the potential impact of COVID-19 on their health as well as their belief that HIV puts them at higher risk for COVID-19 complications; however, only about half of the sample were provided information about how COVID-19 may affect their health which could be a cause of stress in this population. To alleviate this distress, HIV care coordinators should reach out to PLHIV during pandemics to provide them with information and determine what support they may need. Additionally, helping to strengthen HIV selfmanagement skills in anticipation of a pandemic or other crisis could aid in mitigating the negative consequences that were found in this study. One way to do this may be to help foster coping skills in PLHIV. In PLHIV better coping strategies have been associated with improved ART adherence [34]. Furthermore, coping that is specifically focused on HIV self-management has been identified as a predictor for ART adherence [35]. By putting these interventions into place, HIV self-management may be improved for PLHIV during the COVID-19 pandemic and any future pandemics.

This study examined the impact of COVID-19 on HIV self-management, social support, social isolation, depressive symptoms, anxiety, and stress. HIV self-management is essential for successful outcomes and maintaining health in PLHIV [11, 18], which is especially critical during a 
pandemic. We found that overall HIV self-management, depressive symptoms, anxiety, stress, social support, and social networks were all impacted during this time. These findings may have important implications for what aspects of HIV self-management to focus on to best support PLHIV while living through a pandemic.

Supplementary Information The online version contains supplementary material available at https://doi.org/10.1007/s10461-021-03335-4.

Acknowledgements The authors thank the participants for their time in completing this research.

Author Contributions RKW made substantial contributions to: the conception and design of the research; acquisition, analysis, and interpretation of the data; and drafting and revision of the manuscript. WRM made substantial contributions to: the conception and design of the research; interpretation of the data; and drafting and revision of the manuscript.

Funding This study was supported by National Institutes of Health/ National Institute of Nursing Research Grant T32 NR018407.

\section{Declarations}

Conflict of interest The authors report no conflicts of interest.

Ethical Approval This study was determined to be exempt by the Indiana University Institutional Review Board.

\section{References}

1. World Health Organization. Timeline of WHO's response to COVID-19. Geneva: WHO; 2020.

2. Gostin LO, Wiley LF. Governmental public health powers during the COVID-19 pandemic: stay-at-home orders, business closures, and travel restrictions. JAMA. 2020;323(21):2137-8.

3. Banerjee M, Chakraborty S, Pal R. Diabetes self-management amid COVID-19 pandemic. Diabetes Metab Syndr. 2020;14(4):351-4.

4. Druss BG. Addressing the COVID-19 pandemic in populations with serious mental illness. JAMA Psychiat. 2020;77(9):891-2.

5. Helmich RC, Bloem BR. The impact of the COVID-19 pandemic on Parkinson's disease: hidden sorrows and emerging opportunities. J Parkinsons Dis. 2020;10(2):351-4.

6. Kalichman SC, Eaton LA, Berman M, et al. Intersecting pandemics: impact of SARS-CoV-2 (COVID-19) protective behaviors on people living with HIV, Atlanta, Georgia. J Acquir Immune Defic Syndr. 2020;85(1):66-72.

7. Centers for Disease Control and Prevention. People who are at increased risk for severe illness. Atlanta: Centers for Disease Control and Prevention; 2020.

8. Vizcarra P, Pérez-Elías MJ, Quereda C, et al. Description of COVID-19 in HIV-infected individuals: a single-centre, prospective cohort. Lancet HIV. 2020;7(8):e554-64.

9. Gervasoni C, Meraviglia P, Riva A, et al. Clinical features and outcomes of HIV patients with coronavirus disease 2019. Clin Infect Dis. 2020;71(16):2276-8.
10. Del Amo J, Polo R, Moreno S, et al. Incidence and severity of COVID-19 in HIV-positive persons receiving antiretroviral therapy: a cohort study. Ann Intern Med. 2020;173(7):536-41.

11. HIV.gov. HIV care continuum. 2020. https://www.hiv.gov/feder al-response/policies-issues/hiv-aids-care-continuum. Accessed on 28 July 2020.

12. Iribarren S, Siegel K, Hirshfield S, et al. Self-management strategies for coping with adverse symptoms in persons living with HIV with HIV associated non-AIDS conditions. AIDS Behav. 2018;22(1):297-307.

13. Schnall R, Wantland D, Velez O, Cato K, Jia H. Feasibility testing of a web-based symptom self-management system for persons living with HIV. J Assoc Nurses AIDS Care. 2014;25(4):364-71.

14. Webel AR, Asher A, Cuca Y, et al. Measuring HIV self-management in women living with HIV/AIDS: a psychometric evaluation study of the HIV Self-management Scale. J Acquir Immune Defic Syndr. 2012;60(3):e72-81.

15. Algarin AB, Varas-Rodríguez E, Valdivia C, et al. Symptoms, stress, and HIV-related care among older people living with HIV during the COVID-19 pandemic, Miami, Florida. AIDS Behav. 2020;24(8):2236-8.

16. Santos G-M, Ackerman B, Rao A, et al. Economic, mental health, HIV prevention and HIV treatment impacts of COVID19 and the COVID-19 response on a global sample of cisgender gay men and other men who have sex with men. AIDS Behav. 2020;25(2):311-21.

17. Chenneville T, Gabbidon K, Hanson P, Holyfield C. The impact of COVID-19 on HIV treatment and research: a call to action. Int J Environ Res Public Health. 2020;17(12):4548.

18. Webel AR, Sattar A, Schreiner N, Kinley B, Moore SM, Salata RA. The impact of mental wellness on HIV self-management. J Assoc Nurses AIDS Care. 2016;27(4):468-75.

19. Pilkonis PA, Choi SW, Reise SP, et al. Item banks for measuring emotional distress from the Patient-Reported Outcomes Measurement Information System (PROMIS®): depression, anxiety, and anger. Assessment. 2011;18(3):263-83.

20. Salsman JM, Butt Z, Pilkonis PA, et al. Emotion assessment using the NIH Toolbox. Neurology. 2013;80(11 Suppl 3):S76-86.

21. United States Census Bureau. 2010 census regions and divisions of the United States. Suitland: United States Census Bureau; 2010.

22. Heissel A, Zech P, Rapp MA, et al. Effects of exercise on depression and anxiety in persons living with HIV: a meta-analysis. J Psychosom Res. 2019;126:109823-920.

23. Oliveira VHF, Rosa FT, Santos JC, et al. Effects of a combined exercise training program on health indicators and quality of life of people living with HIV: a randomized clinical trial. AIDS Behav. 2020;24(5):1531-41.

24. Bhochhibhoya A, Qiao S, Mao Y, et al. Physical activity, depression, and antiretroviral therapy adherence among people living with HIV: a mediation analysis. J Assoc Nurses AIDS Care. 2019;30(5):556-62.

25. Downing MJ, Millar BM, Hirshfield S. Changes in sleep quality and associated health outcomes among gay and bisexual men living with HIV. Behav Sleep Med. 2020;18(3):406-19.

26. Andini S, Yona S, Waluyo A. Self-efficacy, depression, and adherence to antiretroviral therapy (ART) among Indonesian women with HIV. Enferm Clin. 2019;29(Suppl 2):687-90.

27. Mills JC, Pence BW, Edmonds A, et al. The impact of cumulative depression along the HIV care continuum in women living with HIV during the era of universal antiretroviral treatment. J Acquir Immune Defic Syndr. 2019;82(3):225-33.

28. Wykowski J, Kemp CG, Velloza J, Rao D, Drain PK. Associations between anxiety and adherence to antiretroviral medications in low- and middle-income countries: a systematic review and metaanalysis. AIDS Behav. 2019;23(8):2059-71. 
29. Benoit AC, Burchell AN, O'Brien KK, et al. Examining the association between stress and antiretroviral therapy adherence among women living with HIV in Toronto, Ontario. HIV Res Clin Pract. 2020;21(2-3):45-55.

30. Garrido-Hernansaiz H, Alonso-Tapia J. Social support in newly diagnosed people living with HIV: expectations and satisfaction along time, predictors, and mental health correlates. J Assoc Nurses AIDS Care. 2017;28(6):849-61.

31. Matsumoto S, Yamaoka K, Takahashi K, et al. Social support as a key protective factor against depression in HIV-infected patients: report from large HIV clinics in Hanoi, Vietnam. Sci Rep. 2017;7(1):15489.

32. Chandran A, Benning L, Musci RJ, et al. The longitudinal association between social support on HIV medication adherence and healthcare utilization in the Women's Interagency HIV Study. AIDS Behav. 2019;23(8):2014-24.

33. Kelly JD, Hartman C, Graham J, Kallen MA, Giordano TP. Social support as a predictor of early diagnosis, linkage, retention, and adherence to HIV care: results from the steps study. J Assoc Nurses AIDS Care. 2014;25(5):405-13.

34. Camargo CC, Cavassan NRV, Tasca KI, Meneguin S, Miot HA, Souza LR. Depression and coping are associated with failure of adherence to antiretroviral therapy among people living with HIV/ AIDS. AIDS Res Hum Retroviruses. 2019;35(11-12):1181-8.

35. Vyavaharkar M, Moneyham L, Tavakoli A, et al. Social support, coping, and medication adherence among HIV-positive women with depression living in rural areas of the southeastern United States. AIDS Patient Care STDS. 2007;21(9):667-80.

Publisher's Note Springer Nature remains neutral with regard to jurisdictional claims in published maps and institutional affiliations. 